\title{
Literatur über Städte mit anderen Augen sehen
}

Rezension zu Miles, Malcolm (2018): Cities and Literature. London/New York: Routledge.

Abb. 1 Titel des Buches (Quelle: Routledge Verlag)

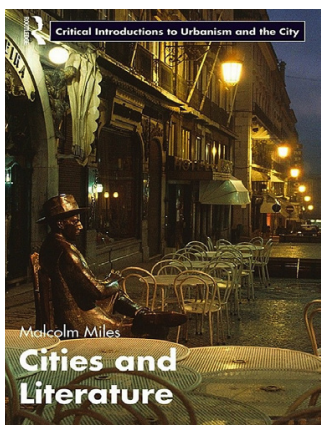

Auf nur 225 Seiten wagt Malcolm Miles gewaltige Sprünge: In seinem 2018 erschienenen Buch Cities and Literature gibt er eine kritische Einführung in die Beziehung zwischen Städten und Literatur und spannt dabei den Bogen vom späten 18. Jahrhundert bis in das 21. Jahrhundert. Dafür nutzt er viele Beispiele aus Europa und Nordamerika, aber auch postkoloniale Literat_innen des Globalen Südens kommen zur Sprache. Sein Verständnis von Literatur ist breit aufgestellt und reicht von Büchern und Aufsätzen über Poesie bis hin zu Postern.

Cities and Literature richtet sich an Sozialwissenschaftler_innen und Literaturbegeisterte, die auf der Suche nach einem neuen Blickwinkel zum Thema Urbanismus sind. Es ist nicht nötig, die besprochenen Bücher zu kennen, wobei dies natürlich hilfreich ist.

Malcolm Miles ist emeritierter Professor für Kulturwissenschaft an der Plymouth University in Großbritannien. Sein Fokus auf den kulturellen Aspekt einer Stadt zieht sich durch das ganze Buch, von Café-concerts in Paris über Weltausstellungen bis hin zu Gedichten. So vielfältig wie die Kultur in einer Stadt ist, so vielfältig sind auch seine literarischen Quellen und deren Bezüge zu Städten. Größen der Literatur wie Henri Lefebvre oder Peter Hall, aber auch Max Weber und Simone de Beauvoir kommen zur Sprache. Am Ende scheinen die Grenzen zwischen Stadt, Kultur und Gesellschaft zu verschwimmen, aber genau das passiert ja auch in Städten.

Das Buch ist eine Liebeserklärung an Literatur über Städte, die so komplex ist wie die Stadt selbst. In zehn Kapiteln geht es unter anderem um die Diskrepanz zwischen der Stadt und dem Land, um Ruinenlandschaften sowie das Konzept ,kosmopolitisch'. Miles nimmt die Lesenden immer wieder an die Hand, indem er historische Übersichten gibt und auch neue Theorien kurz einführt. Er erläutert die zitierte Literatur - manchmal beinahe zu ausführlich - und gibt dazu anschauliche Beispiele in den Zusatzkästen, die jedes Kapitel auflockern.

Das Buch ist nach Themenbereichen geordnet, die in etwa die letzten 250 Jahre der westlichen Geschichte mehr oder weniger chronologisch bearbeiten. Es geht los mit dem Kontrast zwischen Stadt und Land. Danach folgen 
Utopien, Dystopien und Überlegungen zur Metropole. Als nächstes schreibt Miles über Bohemians, Avantgarde-Bewegungen und Ruinenlandschaften. Exil und die Stadt nach dem Zweiten Weltkrieg sind weitere Themen; zuletzt geht es um alternative Städte und den Trend hin zur kosmopolitischen Stadt. Es kommen viele verschiedene Städte als Beispiele zum Einsatz und innerhalb der thematischen Kapitel springt Miles zum Teil zwischen den Jahrhunderten, um Vergleiche zu ziehen.

So gelingt es ihm, Fragen zu behandeln wie etwa die, ob und wie die Repräsentation von Städten in der Literatur urbane Realitäten zeigt und diese vielleicht sogar beeinflussen kann. Er analysiert die Bedeutung von zeitgenössischer Literatur im Hinblick auf die Wahrnehmung von Städten und untersucht zugleich, wie vorherrschende urbane Bedingungen die Literatur beeinflussen.

Es ist möglich, die Kapitel je nach eigener Interessenslage auch selektiv zu lesen, da sie für sich alleinstehen können. Dabei wird deutlich, dass Städte vor allem eines sind: Der wesentliche Schauplatz für moderne Literatur im Zeitalter des Kapitalismus. Literatur drückt somit auch die negativen und positiven Aspekte des kapitalistischen Lebens in der Stadt aus. Besonders eindrucksvoll sind hier die Kapitel zu Dystopie und zu Ruinenlandschaften.

Im Gegensatz zu ähnlichen Werken wie The City in Literature (Lehan 1998) lässt Miles auch postkoloniale Autor_innen zu Wort kommen. Hier sind unter anderem Ngugi wa Thion'go oder Léopold Senghor mit seinen Überlegungen zur Négritude zu nennen. Darüber hinaus legt Miles einen wertvollen Schwerpunkt auf Literatur von Frauen und LGBTQI-Gruppen, indem er beispielsweise die Arbeit von Audre Lorde, Tsitsi Dangarembga oder Gloria Anzaldua diskutiert. Dabei weist er immer wieder darauf hin, welche Autor_innen seiner Meinung nach vernachlässigt werden. Denn an Charles Dickens oder Emile Zola denkt wohl jeder, wenn es um Literatur und Städte geht, doch wer würde darauf kommen, Literatur aus Nairobi, Kritiken von Leonie Sandercock oder Romane von Zadie Smith einzubeziehen? Mit der gelungenen Inklusion trägt Miles wesentlich dazu bei, unser Verständnis von Literatur zu erweitern.

Das Thema Stadt ist derzeit in der Forschung sehr beliebt und Malcolms Werk stellt eine sehr gute Orientierung zum Stellenwert des Urbanen in der Literatur dar. Wie die Stadt selbst ist auch die Literatur über die Stadt nur schwer zusammenzufassen, aber Miles macht einen insgesamt sehr gelungenen Versuch. Jedoch bleibt auch Kritik: Obwohl Miles sich bemüht, viele weibliche, postkoloniale, bisher vernachlässigte Autor_innen einzubeziehen, liegt der Fokus des Werkes dennoch auf den großen westlichen Metropolen: Allen voran London, aber auch Berlin, Paris, Prag, Wien und New York City. Fairerweise gibt Miles das auch von Anfang an zu, wobei nicht ganz klar wird, woher seine Entscheidung kommt, nur postkoloniale Autor_innen zu nennen. Denn auch zu Kolonialzeiten gab es spannende Literatur in den besetzten Ländern.

Besonders interessant ist jedoch das neunte Kapitel, das sich Themen wie ,otherness' und , alternate cities' widmet. Hier geht es mehr um Stadtplanung als in den anderen Teilen des Buches, und neben dem ,Recht auf die Stadt von Henri Lefebvre widmet Miles sich auch der literarischen Darstellung von postkolonialen Städten wie Nairobi. Multikulturelle Städte, das Ideal der Partizipation und die Frage, was eine Nachbarschaft eigentlich ausmacht, 
werden angerissen und machen Lust, sich noch mehr mit diesen Themen zu beschäftigen. Interessant ist auch, dass sich der häufig despektierlich gebrauchte Begriff ,Slum` im ganzen Buch nicht einmal finden lässt.

Trotz der großen Vielfalt an Themen und der Zeitreise von der Industrialisierung bis hin in die Postmoderne gelingt es Malcolm Miles, den roten Faden zu behalten. Dabei fehlt mir jedoch ein wichtiges Thema, das in einem Buch aus dem Jahr 2018 eigentlich vorhanden sein sollte: Der Klimawandel. Obwohl Malcom Miles auf das Verhältnis zwischen Stadt und Land sowie Umwelt-Dystopien eingeht, wäre es interessant gewesen, das Thema Klimawandel im Bezug auf die Stadt ausführlich zu erläutern. Es wäre wünschenswert, aktuelle Werke wie etwa $Y$ los mariachis callaron (Und die Mariachis schwiegen) von Rivera Calderón (2018) aufzunehmen, um zum Beispiel darzustellen, wie Mexiko-Stadt - die größte Metropole der westlichen Hemisphäre - dem Klimawandel ausgeliefert ist.

Die Lektüre ist erfrischend, intellektuell herausfordernd und bereichernd. Jedoch wäre ein ausführlicheres abschließendes Kapitel wünschenswert, um die offenen und aufgeworfenen Fragen wie etwa die nach dem Zusammenspiel von Stadt und Land oder nach der Rolle von Frauen in der Literatur über Städte noch einmal zusammenzubringen. Eine wichtige Frage beantwortet Miles allerdings schon selbst:

„Writing, as the high culture called literature, or in mass-market fiction, film and television scripts, does not provide answers, nor propel humanity along a given trajectory, because the future is produced through interactions of human and nun-human (environmental) agencies, and the process goes on. [...] The urban imaginary is held in tension by such polarities, its work done between images of what was, what is and what might be.“(Miles 2018: 225).

Damit macht er deutlich, dass Literatur über Städte viele Funktionen und Perspektiven einnimmt, aber letztendlich nur Ideen und Vorstellungen bietet. Sie kann keine eindeutige Definition von Stadt liefern, da dieses Konzept zu komplex ist. Letztendlich spiegelt das Buch sehr gut wider, was auch eine Stadt zu tun vermag: Es bietet eine Fülle von Aspekten, von denen sich jede_r diejenigen herauspicken kann, die ihm oder ihr am besten gefallen. Dicht, informativ, bunt und vielfältig - Cities and Literature ermutigt alle Sozialwissenschaftler_innen sowie andere Interessierte, in Zukunft mehr auf die Darstellung von Städten in der Literatur zu achten. Für dieStadtforschung lässt sich lernen, dass die Repräsentation von Städten beinahe ubiquitär ist, was zum Beispiel interessant für historische und futuristische Vergleiche ist. Praktische ,Anleitungen ' für die Stadtplanung jedoch bietet dieses Buch nicht. Es ist daher besonders gut für Sozialwissenschaftler_innen und für transdisziplinäre Vorhaben geeignet. 


\section{Autor_innen}

Laura von Puttkamer lebt derzeit in Mexiko-Stadt und arbeitet als freiberufliche Autorin, Bloggerin (parCitypatory.org) und Journalistin. Ihr Schwerpunkt liegt auf Partizipation in der Stadtentwicklung im Globalen Süden.

lauravonputtkamer@gmail.com

\section{Literatur}

Lehan, Richard (1998): The City in Literature. An Intellectual and Cultural History. Berkeley: University of California Press.

Miles, Malcolm (2018): Cities and Literature. London and New York: Routledge.

Rivera Calderón, Fernando (2018): Y los mariachis callaron. Ciudad de México: Reservoir Books. 